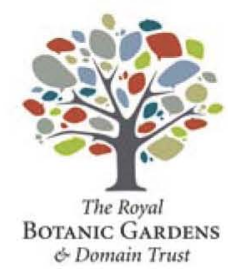

\title{
MOSSES AND THEIR DISTRIBUTION IN THE AUSTRALIAN CAPITAL TERRITORY
}

\author{
Helen P. Ramsay' \& H. StreimanN2 ${ }^{2}$
}

(Accepted for publication 29.3.1983)

\begin{abstract}
Ramsay, Helen P.' \& Streimann, H.? ('School of Botany, University of New South Wales, Kensington, N.S.W. Australia 2033; ${ }^{2}$ Herbarium, National Botanic Gardens, Canberra A.C.T. Australia 2601). Telopea 2(5): 559-574. - The Australian Capital Territory is geographically located on the Southern Tablelands of New South Wales. The area is physiographically and climatically diverse with alluvial terrain to high ridges reaching $1900 \mathrm{~m}$. More than 180 species from 39 families and their distribution within the A.C.T. are recorded. Data on distribution in other regions of N.S.W. and in other Australian states are also given. Affinities are mainly with temperate mosses of southern Australia.
\end{abstract}

\section{INTRODUCTION}

The Australian Capital Territory (A.C.T.), although a discrete political territory, is geographically located on the Southern Tablelands of New South Wales. In area it covers $2357 \mathrm{sq}$. $\mathrm{km}$ with more than half being occupied by rugged mountainous catchment areas (Learmonth 1973). Canberra, the capital city of Australia, lies on a plain $600 \mathrm{~m}$ above sea level and approximately $100 \mathrm{~km}$ from the coast on the western side of the Great Dividing Range in the A.C.T.

The physiography of the A.C.T. can be divided into: (i) high ridges and steep hillslopes; (ii) rolling terrain; (iii) gently undulating terrain; (iv) alluvial terrain. The Murrumbidgee River and its tributaries, the Molonglo and Cotter rivers, pass through the Territory. The Murrumbidgee and Molonglo drain from the Great Dividing Range, while the Cotter originates in the ranges west of Canberra. On the western boundary are the Brindabella-Bimberi Ranges that connect with the Snowy massif. The Scabby-Boboyan Ranges form the southern boundary. A number of peaks within these ranges reach $1800 \mathrm{~m}$ with Bimberi Peak $1903 \mathrm{~m}$ in the south the highest. They form a dissected plateau, part of the Kosciusko peneplain, cut across by folded and steeply dipping Ordovician and Silurian slates, schists, slaty shales and grit, Silurian volcanics, with some Palaeozoic porphyry, granite, and Devonian igneous rocks. Alluvial flats form the Canberra basin, a rain-shadow area often having less than $600 \mathrm{~mm}$ rainfall (Learmonth 1973).

Great variations in climate occur between the Canberra plain and the higher peaks on the western ranges. There are numerous rain-shadows in the deeper valleys, but the main rain-shadow is the Canberra plain itself. It is dry during the summer, most of the rainfall being derived from a few downpours, but high evaporation rates, about $1600 \mathrm{~mm}$ annually, reduce its effectiveness. On the ranges the precipitation may reach $1500 \mathrm{~mm}$ per year, and is more uniform. In winter, inversions cause fogs and some of the grasslands in the higher valleys may have evolved from the settling of cold air. In addition, Canberra gets about 100 days of frost per annum, with a large range of diurnal temperatures in both winter and summer. Less is known of the climate at higher altitudes but data in Table 1 (see page 563) illustrate some of the variation at different altitudes.

Snow occurs on the ranges above $1220 \mathrm{~m}$ and on the higher parts (above $1500 \mathrm{~m}$ ), where it may lie for 3 to 4 months. Soils tend to correlate to topography, geology and the climatic influences over long periods. 


\section{DISTRIBUTION WITHIN THE A.C.T.}

Vegetation in the A.C.T. has affinities with elements from Mt Kosciusko, the Victorian Alps and Tasmania. The zonations of the A.C.T. vascular flora are mostly altitudinal. Little has yet been done on the altitudinal distribution or the ecology of the non-vascular flora except on Mt Ainslie and Black Mountain. Much of the vegetation has been greatly modified by man since European settlement (Pryor 1954).

Natural features or major roads have been used to define five topographically separate divisions of the A.C.T. for this project (see Fig. 1). The Brindabella Ranges division encompasses almost all the topographical environments found in the A.C.T. The following are short descriptions of each division. (Jervis Bay is excluded as it is geographically in a separate part of New South Wales and unrelated floristically to the rest of the A.C.T.)

Division 1. BOOTH RANGE (east side of Gudgenby Road)

Three vegetation types predominate here. At higher altitudes $(>700 \mathrm{~m})$ Eucalyptus delegatensis - E. dalrympleana form wet sclerophyll forest. In the broad upper valley systems $E$. pauciflora - E. stellulata savannah woodlands are present, while in the northern area between the Booth and Clear Ranges E. melliodora - E. blakelyi woodlands occur (Burbidge \& Gray 1970). There has been very little disturbance to the area except for grazing in the north. Most of the area is in the Namadgi National Park.

The altitude ranges from $640 \mathrm{~m}$ to $1600 \mathrm{~m}$ (Mt Clear) with foliated granodiorite as the predominant rock.

Division 2. SOUTHERN RANGES (south of Orroral and west of Gudgenby road) The vegetation comprises mostly Eucalyptus delegatensis $-E$. dalrympleana wet sclerophyll forest with scattered pockets of $E$. pauciflora $-E$. stellulata savannah woodlands along the larger stream valleys. The higher ranges have alpine woodlands of stunted E. pauciflora and grasses (Burbidge \& Gray 1970). Small areas of Sphagnum swamp are scattered throughout this region. Least disturbance has taken place in this division. The Namadgi National Park includes a large area here as well as Division 1.

The main rocks are foliated granodiorites that form large spectacular outcrops. Along the Cotter Valley, from near the confluence of the Orroral and Gudgenby rivers to the southern border, sandstone and greywacke are found. The altitude ranges from $840 \mathrm{~m}$ to $1903 \mathrm{~m}$ (Bimberi Peak, the highest peak in the A.C.T.).

Division 3. BRINDABELLA RANGES (north of the Orroral-Cotter Hut roads) The vegetation in this division varies greatly from tree-fern-shaded, humid gullies at the Tidbinbilla Nature Reserve to alpine woodlands of Eucalyptus pauciflora on the higher parts of the ranges adjacent to the N.S.W. border. The undulating lowlands along the Murrumbidgee and Paddys rivers carries E. melliodora $E$. blakelyi savannah woodland with Casuarina cunninghamii along the banks. However, much of this is now grazing land or Pinus radiata plantations. Eucalyptus macrorhyncha - E. rossii dry sclerophyll forest occurs on northerly and westerly faces of the mountains. Also a section of $E$. fastigata $-E$. viminalis wet sclerophyll forest occurs along the Cotter Valley with a scattered dense understorey of Compositae, Myrtaceae and Rhamnaceae (Burbidge \& Gray 1970). Extensive, scattered Sphagnum swamps occur at high altitudes on tributaries of the Cotter River. Within the area, moist gullies with tree-ferns and numerous "coastal" moss species, e.g. Camptochaete and Hypnodendron, are present.

Most of this area, including Mt Gingera, is foliated granodiorite, although a section of greywacke, slate and sandstone occurs at Mts Aggie, Franklin and Ginini. Mt Coree is covered by volcanic rocks and part of the Tidbinbilla Range is 


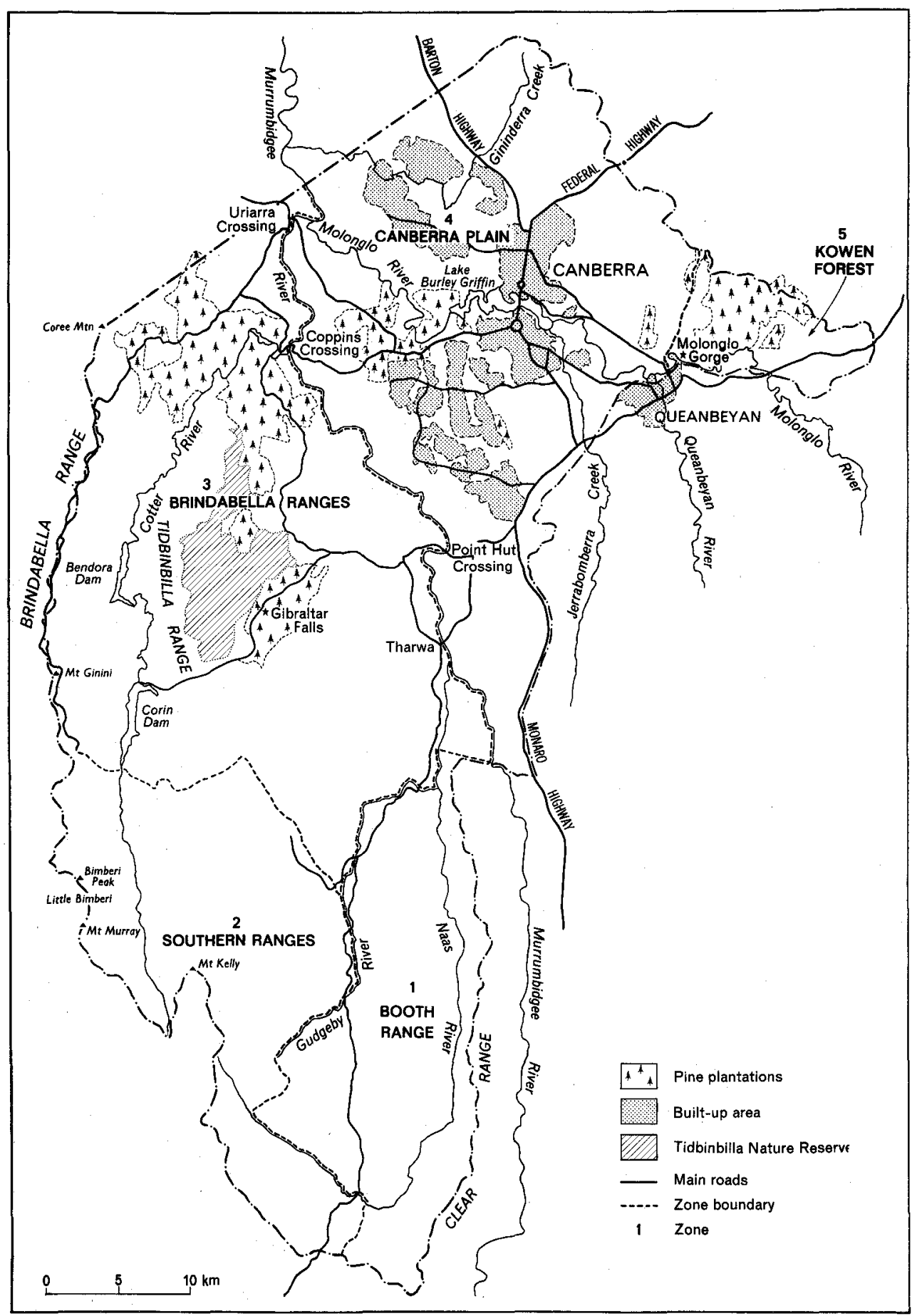

Fig. 1. Australian Capital Territory showing division into areas defined for this study. 
quartzite. Volcanic rocks cover extensive areas in the north. The altitude varies between 410 and $1856 \mathrm{~m}$ (Mt Gingera).

Division 4. CANBERRA PLAIN (bounded on south-east by Sutton Road and west by the Murrumbidgee River)

This plain has several outcropping mountains - Black Mountain, Mts Majura and Ainslie, and Red Hill. The area is predominantly a mixture of acid volcanic rocks and porphyry. The altitude varies between 410 and $890 \mathrm{~m}$ (Mt Majura). The plain is drained by the Molonglo and Murrumbidgee rivers while most of the smaller streams are seasonal.

Much disturbance has been caused by grazing and urbanization. As a result, a large proportion of the original Themeda-Poa grasslands of the river flats and the Eucalyptus melliodora - E. blakelyi savannah woodlands now occur only in small pockets. There are small sections of $E$. rossii $-E$. macrorhyncha, with the most extensive being around Black Mountain.

Division 5. KOWEN FOREST (east of Sutton Road)

Eucalyptus rossii - E. macrorhyncha dry sclerophyll forest predominates and savannah woodlands occur along the northern boundary of the A.C.T. Extensive Pinus radiata plantations occur in this division.

Folded sedimentary rocks (greywacke, slate and sandstone) form the underlying strata that generally developed shallow, gravelly soils. Most of the area is hilly or rolling country with a steep section along a fault line near the Sutton Road. Further steep sections are encountered along the Molonglo River. This is now a nature reserve and a popular recreation area. The altitude varies between 610 and $920 \mathrm{~m}$.

\section{COMPILATION OF DATA}

The following data, presented in Table 2, have been compiled from field work and herbarium specimens held in the Herbarium Australiense (CANB), and the Herbarium, National Botanic Gardens (CBG) in Canberra; the National Herbarium of New South Wales (NSW), the Herbarium, University of New South Wales (UNSW), the Ray Herbarium, University of Sydney (SYD) and the private collection of Professor D.G. Catcheside.

Names follow those in use according to Index Muscorum plus supplements (Wijk et al. 1959-1969; Crosby 1977, 1979; Crosby \& Bauer 1981) together with information from Scott \& Stone (1976), Catcheside (1980) and other taxonomic publications. For synonyms, nomina nuda and further information see Census of New South Wales mosses (Ramsay 1984). Families are arranged according to Crosby \& Magill (1981), with genera and species listed alphabetically in families.

\section{KEY TO SYMBOLS USED FOR DISTRIBUTION}

LHI Lord Howe Island

C Coast

$T$ Tablelands

WS Western Slopes

WP Western Plains
FWP Far Western Plains

$N$ North

C Central

$S$ South

NSW New South Wales

- $>3$ collections in the subdivision

0 no sighted or cited specimen from the subdivision

? specimen or cited locality inadequate for reliable record

$*$ number of specimens $\leqslant 3$

$\mathbf{Q}-$ Queensland, $\mathbf{V}-$ Victoria, $\mathbf{T}-$ Tasmania, $\mathbf{Y}-$ Northern Territory,

S - South Australia, w - Western Australia 
TABLE 1. CLIMATIC DATA FOR CANBERRA AND BULLS HEAD.

\begin{tabular}{|c|c|c|c|c|c|c|c|c|}
\hline \multirow{3}{*}{$\begin{array}{l}\text { Location } \\
\therefore\end{array}$} & \multirow{3}{*}{$\begin{array}{c}\text { Alt } \\
\text { (m) }\end{array}$} & \multicolumn{6}{|c|}{ Temperature $\left({ }^{\circ} \mathrm{C}\right)$} & \multirow{3}{*}{$\begin{array}{l}\text { Rain } \text { Evapn }^{n} \\
\text { Av. annual (mm) }\end{array}$} \\
\hline & & \multicolumn{2}{|c|}{ Jan. } & \multicolumn{2}{|c|}{ July } & \multicolumn{2}{|c|}{ Extremes } & \\
\hline & & $\begin{array}{l}\text { Av. } \\
\text { max. }\end{array}$ & $\begin{array}{l}\text { Av. } \\
\text { min. }\end{array}$ & $\begin{array}{l}\text { Av. } \\
\text { max. }\end{array}$ & $\begin{array}{l}\text { Av. } \\
\text { min. }\end{array}$ & Max. & Min. & \\
\hline Canberra & 600 & 27.5 & 13.0 & 11.1 & -0.4 & 42.2 & -10.0 & 1600 \\
\hline Bulls Head* & 1320 & 21.3 & 9.7 & 5.2 & -2.0 & 35.1 & -12.2 & 1009 \\
\hline
\end{tabular}

*Junction Brindabella and Bendora Dam roads.

TABLE 2. MOSSES AND THEIR DISTRIBUTION IN THE A.C.T.

(families arranged according to Crosby \& Magill 1981)

\begin{tabular}{|c|c|c|c|c|c|c|c|c|}
\hline \multirow{3}{*}{\multicolumn{2}{|c|}{ Name }} & \multicolumn{7}{|c|}{ Distribution } \\
\hline & & \multicolumn{5}{|c|}{ A. C. T. } & \multirow[b]{2}{*}{ N.S.W. } & \multirow{2}{*}{$\begin{array}{l}\text { OTHER } \\
\text { STATES }\end{array}$} \\
\hline & & $\stackrel{l}{\text { Booth Ra. }}$ & S. Ranges & $\stackrel{3}{\text { Brindabella }}$ & $\stackrel{4}{\text { Canberra }}$ & $\stackrel{5}{\text { Kowen }}$ & & \\
\hline 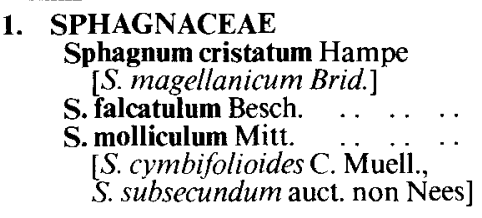 & \begin{tabular}{ll|} 
& \\
$\cdots$ & $\cdots$ \\
$\cdots$ & $\cdots$ \\
& $\cdots$
\end{tabular} & $\begin{array}{l}\star \\
0 \\
0\end{array}$ & $\begin{array}{l}\bullet \\
\star \\
\star\end{array}$ & $\begin{array}{l}\star \\
\star \\
0\end{array}$ & $\begin{array}{l}0 \\
0 \\
0\end{array}$ & $\begin{array}{l}0 \\
0 \\
0\end{array}$ & $\begin{array}{l}\text { NCCCT } \\
\text { ST only } \\
\text { CCT ST }\end{array}$ & $\begin{array}{l}\text { QVT } \\
\text { QVT } \\
\text { VTSW }\end{array}$ \\
\hline $\begin{array}{l}\text { 2. ANDREAEACEAE } \\
\text { Andreaea australis Mitt. } \\
\text { A. mutabilis Hook. f. \& Wils. } \\
\text { [A. rupestris auct. non Hedw.] } \\
\begin{array}{l}\text { A. nitida Hook. f. \& Wils. } \\
\text {. . }\end{array}\end{array}$ & \begin{tabular}{ll|} 
& \\
$\cdots$ & $\cdots$ \\
$\cdots$ & $\cdots$ \\
& $\ldots$
\end{tabular} & $\begin{array}{l}0 \\
\star \\
0\end{array}$ & $\begin{array}{l}0 \\
0 \\
0\end{array}$ & $\begin{array}{l}\star \\
\star \\
\star\end{array}$ & $\begin{array}{l}0 \\
0 \\
0\end{array}$ & $\begin{array}{l}0 \\
0 \\
0\end{array}$ & $\begin{array}{l}\text { ST only } \\
\text { CT ST } \\
\text { ST only }\end{array}$ & $\begin{array}{l}\text { VT } \\
\text { VT } \\
\text { VT }\end{array}$ \\
\hline
\end{tabular}

$0-$ not collected $\quad \star \leqslant 3$ collections

- $>3$ collections 


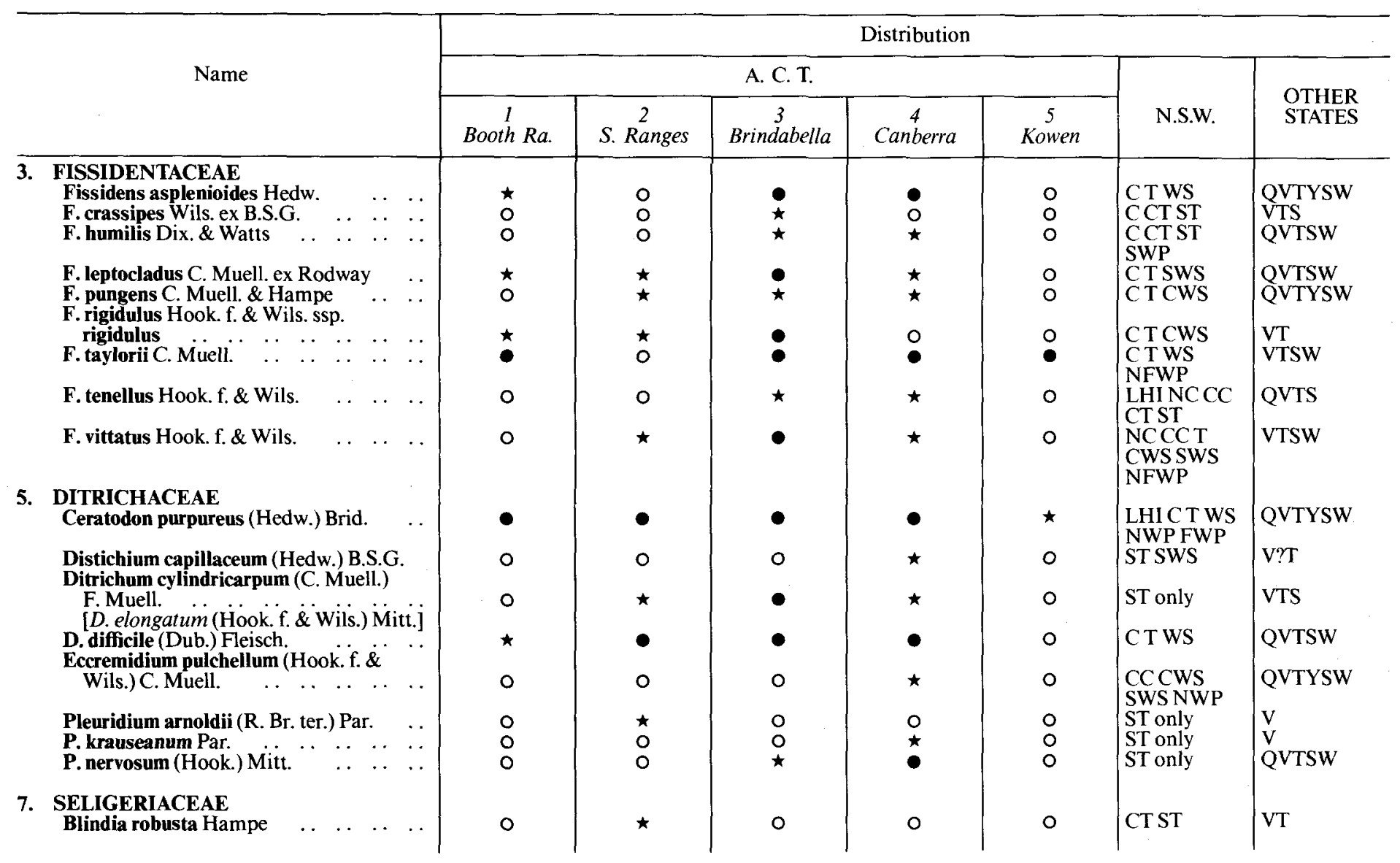


8. DICRANACEAE Campylopus austro-subulatus Broth. \&

C. clavatus (R. Br.) Wils

C. introflexus (Hedw.) Brid.

C. pallidus Hook. f. \& Wils

†Dicnemoloma pallidum (Hook.) Wijk \& Marg.

Dicranoloma billardieri (Brid.) Par.

D. dicarpum (Nees) Par.

D. robustum (Hook. f. \& Wils.) Par. var. setosum (Hook. f. \& Wils.) Sainsb.

Trematodon amoenum (C. Muell.) Stone $\&$ Scott

T. flexipes Mitt.

mackayi (R. Br. ter) Brot

13. ENCALYPTACEAE

Encalypta vulgaris Hedw.

14. POTTIACEAE

Acaulon integrifolium C. Muell. .. .

Barbula calycina Schwaegr.

[Tortella calycina (Schwaegr.) Dix.]

B. crinita Schultz , . . . .

B. hornschuchiana Schultz _. . . . $\begin{array}{llllll}\text { B. torquata Tayl. } \neq \quad \ldots & \ldots & \ldots & \ldots & \ldots\end{array}$

B. unguiculata Hedw.

Bryoerythrophyllum jamesonii (Tayl.)

Crum

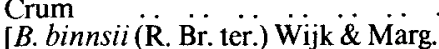

Desmatodon convolutus (Brid.) Grout

D. recurvatus (Hook.) Mitt.

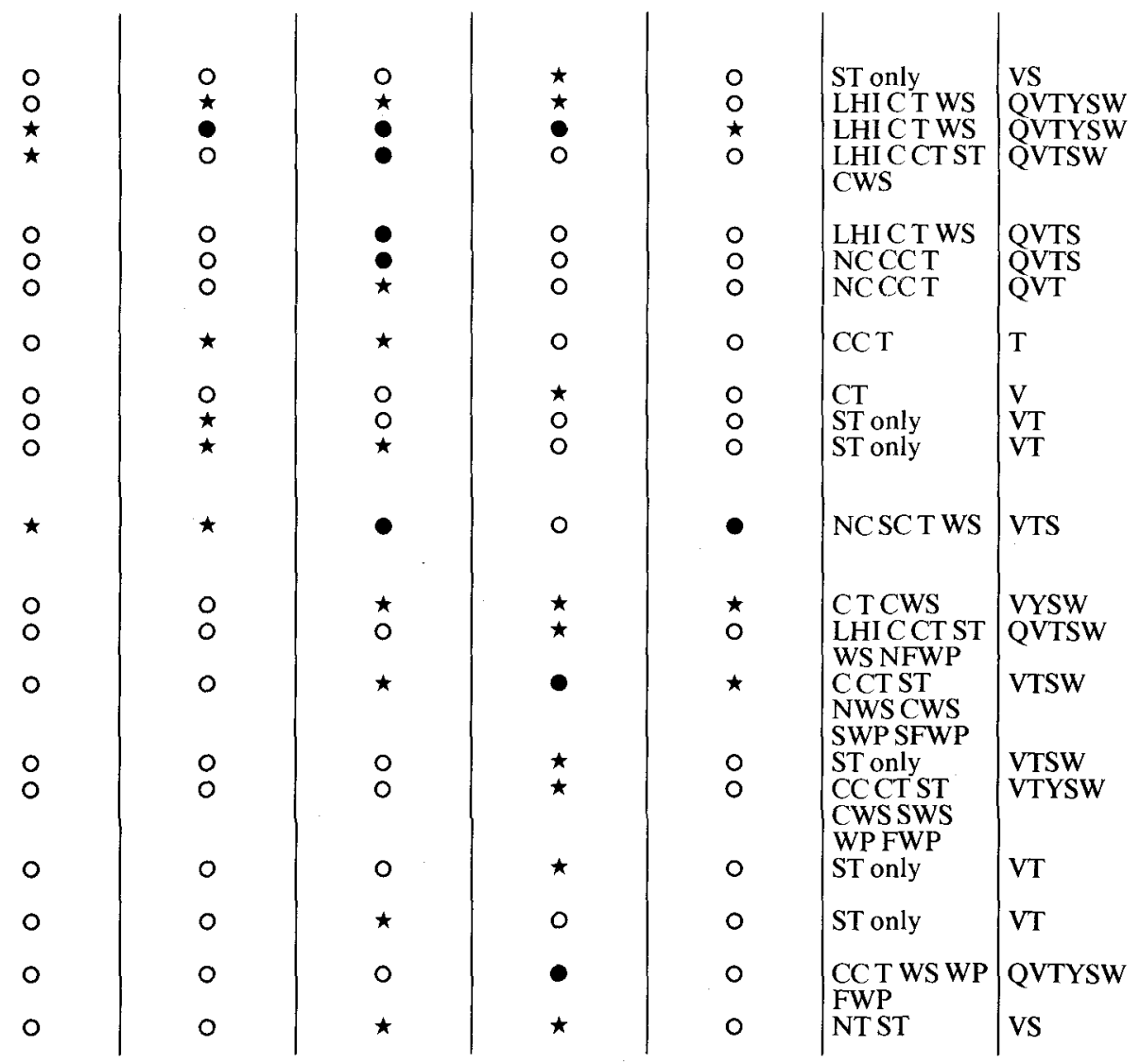

$\dagger$ Retained here as Dicnemoloma although Crosby (pers. comm.) and Crosby \& Magill (1981) point out this name is preceded by Sclerodontium.

$\ddagger=$ Didymodon in Catcheside (1981). 


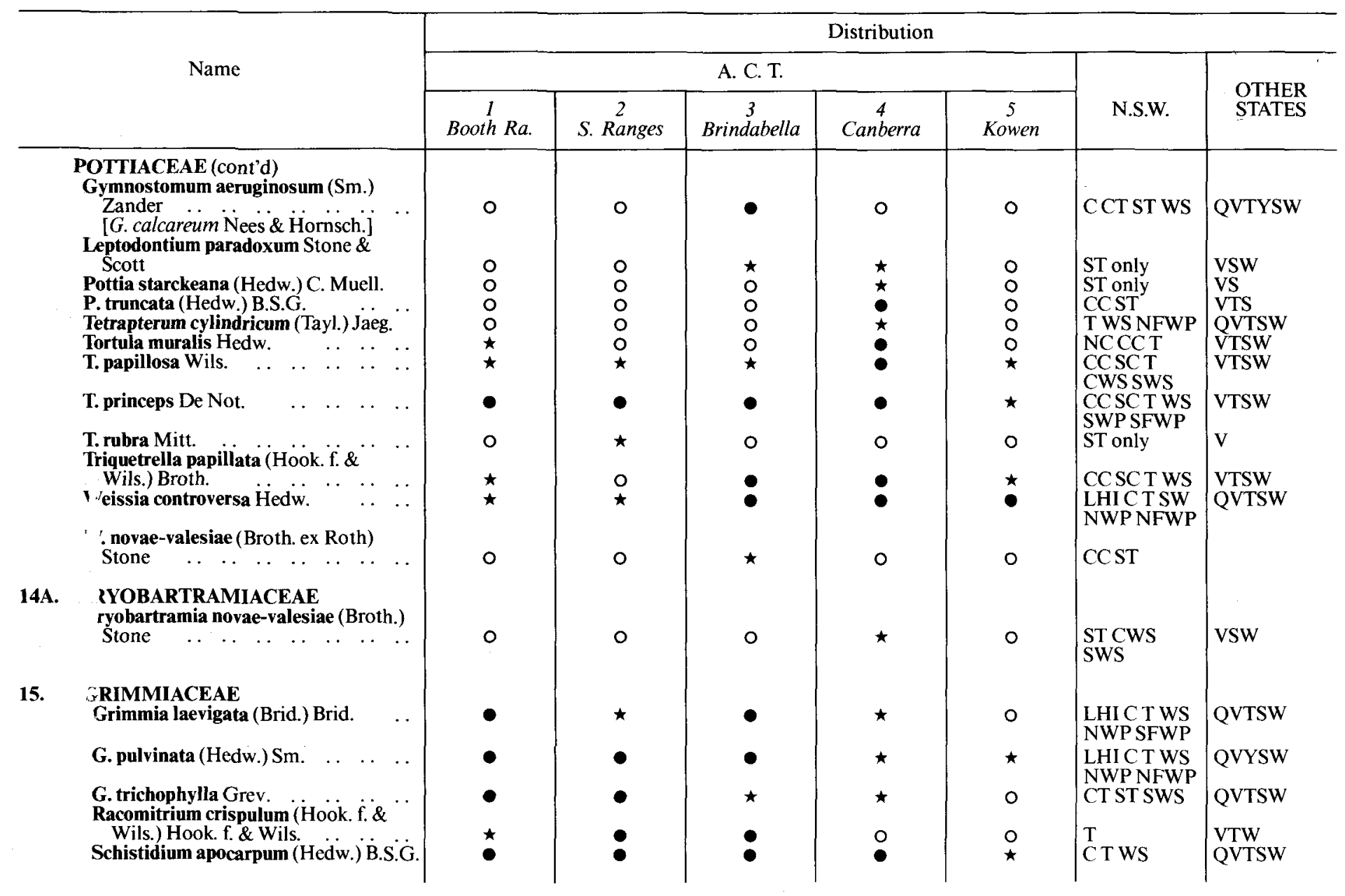


16. GIGASPERMACEAE

Gigaspermum repens (Hook.) Lindb.

19. FUNARIACEAE

Funaria apophysata (Tayl.) Broth. . .

$\begin{array}{lllllllll}\text { F. glabra Tayl. } & \ldots & \ldots & \ldots & \ldots & \ldots & \ldots\end{array}$

F. gracilis (Hook. f. \& Wils.) Broth.

F. hygrometrica Hedw. .. $\quad \ldots \quad \ldots$

Goniomitrium acuminatum Hook. \&$$
\text { Wils. }
$$

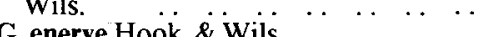

Physcomitrium pyriforme Brid. . . . [P. conicum Mitt.]

21. SPLACHNACEAE

Tayloria octoblepharum (Hook.) Mitt.

\section{BRYACEAE}

Brachymenium coarctum Bosch \& Lac. B. exile (Dozy \& Molk.) Bosch \& Lac. B. cf. preissianum (Hampe) Jaeg.

Bryum $c f$. altisetum C. Muell.

B. apiculatum Schwaegr.

[Ochi (pers. comm.) identified A.C.T. specimens as $B$. apiculatum and put $B$. nitens into synonymy: the former name is not used in main census.]

B. argenteum Hedw.

B. $c f$. australe Hampe

B. billardieri Schwaegr. var. billardieri

B. blandum Hook. f. \& Wils. var.

blandum

B. caespiticium Hedw. $\quad \ldots \quad$.

\begin{tabular}{|c|c|c|c|c|c|c|}
\hline 0 & $\star$ & $\star$ & $\star$ & 0 & $\begin{array}{l}\text { CC SCT } \\
\text { NWS CWS } \\
\text { WP NFWP }\end{array}$ & QVTYSW \\
\hline 0 & 0 & $\star$ & $\star$ & $\star$ & $\begin{array}{l}\text { CCT WS } \\
\text { NFWP }\end{array}$ & QVTYSW \\
\hline 0 & 0 & $\star$ & 0 & 0 & $\begin{array}{l}\text { CTWS } \\
\text { NFWP }\end{array}$ & QVTYSW \\
\hline 0 & 0 & $\star$ & $\star$ & 0 & $\begin{array}{l}\text { NCCC ST } \\
\text { NWS SWS }\end{array}$ & VTYSW \\
\hline 0 & 0 & $\star$ & - & $\star$ & $\begin{array}{l}\text { LHI CT } \\
\text { NWS SWS } \\
\text { CWS SFWP }\end{array}$ & QVTYSW \\
\hline $\begin{array}{l}0 \\
0\end{array}$ & $\begin{array}{l}0 \\
0\end{array}$ & $\begin{array}{l}0 \\
0\end{array}$ & 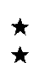 & $\begin{array}{l}0 \\
0\end{array}$ & $\begin{array}{l}\text { CCST CWS } \\
\text { CCNTST }\end{array}$ & $\begin{array}{l}\text { QYSW } \\
\text { VSW }\end{array}$ \\
\hline 0 & 0 & $\star$ & 0 & 0 & $\begin{array}{l}\text { NWS CWS } \\
\text { NWP NFWP } \\
\text { CCST }\end{array}$ & VT \\
\hline $\mathrm{O}$ & $\star$ & - & $\star$ & $\star$ & $\mathrm{CT}$ & QVTSW \\
\hline 0 & 0 & 0 & $\star$ & 0 & $\mathrm{NCCC}$ & $\mathrm{T}$ \\
\hline $\mathrm{O}$ & 0 & 0 & $\star$ & $\star$ & ST only & \\
\hline 0 & 0 & 0 & $\star$ & 0 & $\mathrm{CC}$ & QVTYSW \\
\hline 0 & 0 & $\star$ & $\star$ & 0 & ST only & VA \\
\hline 0 & 0 & $\star$ & $\star$ & 0 & $\begin{array}{l}\text { NCCCCT } \\
\text { ST }\end{array}$ & $\mathrm{Q}$ \\
\hline$\star$ & $\star$ & $\bullet$ & - & 0 & $\begin{array}{l}\text { LHI C T WS } \\
\text { FWP }\end{array}$ & QVTYSW \\
\hline$\star$ & 0 & $\star$ & 0 & 0 & $\mathrm{CCST}$ & VT \\
\hline 0 & $\bullet$ & - & 0 & - & LHI C T WS & QVTSW \\
\hline$\star$ & 0 & $\star$ & 0 & 0 & CT ST & VT \\
\hline 0 & 0 & $\star$ & 0 & 0 & $\begin{array}{l}\text { CCCT ST } \\
\text { NWS }\end{array}$ & VTS \\
\hline
\end{tabular}




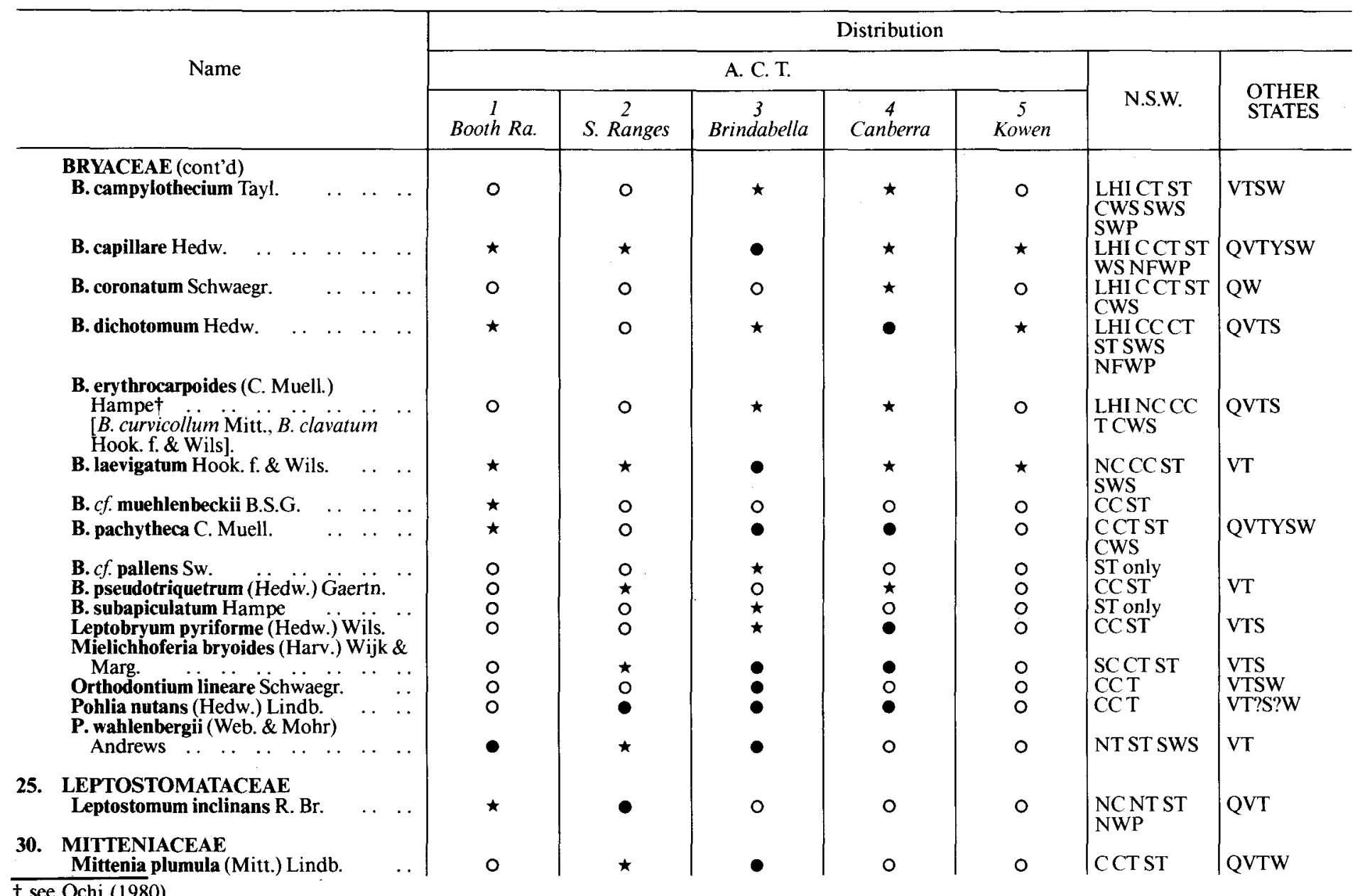


32. RHIZOGONIACEAE

Leptotheca gaudichaudii Schwaegr.

Pyrrhobryum mnioides (Hook.) Wils.

Rhizogonium bifarium (Hook.) Schimp.

R. distichum (Sw.) Brid.

R. novae-hollandiae (Brid.) Brid.

33. HYPNODENDRACEAE

Hypnodendron vitiense Mitt. ssp. australe Touw

34. AULACOMNIACEAE

Aulacomnium palustre (Hedw.) Schwaegr.

35. MEESIACEAE

Meesia muelleri C. Muell. \& Hampe

M. triquetra (L.) Aongstr.

37. BARTRAMIACEAE

Bartramia hampei (Mitt.) Catcheside

B. papillata Hook. f. \& Wils.

B. aff. stricta Brid.

Breutelia affinis (Hook.) Mitt.

B. pendula (Sm.) Mitt.

Conostomum curvirostre (Mitt.) Mitt.

C. pentastichum (Brid.) Lindb.

C. pusillum Hook f. \& Wils.

Philonotis scabrifolia (Hook. f. \& Wils.)

Braithw.

$\begin{array}{llll}\text { P. } c f \text {. tenuis (Tayl. } \text { ) Reichdt. } & \ldots & \ldots & \ldots\end{array}$

41. PTYCHOMITRIACEAE

Ptychomitrium australe (Hampe) Jaeg.

42. ORTHOTRICHACEAE

Macromitrium ligulare Mitt.

Orthotrichum cupulatum Hoffm

O. longithecum $\mathrm{R}$. Br. ter.

\begin{tabular}{|c|c|c|c|c|c|c|}
\hline 0 & - & - & 0 & 0 & SC T CWS & QVT \\
\hline$\star$ & - & - & 0 & 0 & CT & QVT \\
\hline$\star$ & $\star$ & 0 & 0 & 0 & CCNT ST & VT \\
\hline 0 & 0 & $\star$ & 0 & 0 & $\begin{array}{l}\text { NCCC CT } \\
\text { ST }\end{array}$ & QVT \\
\hline 0 & 0 & $\star$ & 0 & 0 & $\begin{array}{l}\text { NC CC CT } \\
\text { ST }\end{array}$ & VT \\
\hline $\mathrm{O}$ & $\star$ & - & 0 & 0 & CT SWS & QVT \\
\hline 0 & - & $\star$ & $\star$ & 0 & NCST & VT \\
\hline た & $?$ & $?$ & $\begin{array}{l}0 \\
0\end{array}$ & $\begin{array}{l}0 \\
0\end{array}$ & $\begin{array}{l}\text { ST only } \\
\text { ST only }\end{array}$ & $\begin{array}{l}\text { V } \\
\text { V }\end{array}$ \\
\hline 0 & 0 & 0 & - & 0 & NT ST CWS & VTW \\
\hline$\star$ & - & - & 0 & 6 & CC T WS & VTSW \\
\hline 0 & 0 & $\star$ & 0 & 0 & $\begin{array}{l}\text { CCT NWS } \\
\text { SWS }\end{array}$ & QVTSW \\
\hline$\star$ & $\star$ & - & - & - & CCSC T WS & VTSW \\
\hline$\star$ & - & - & $\star$ & 0 & CT NWS & VTW \\
\hline 0 & $\star$ & $\star$ & 0 & 0 & ST only & \\
\hline 0 & $\star$ & 0 & 0 & 0 & ST only & VT \\
\hline $\mathrm{O}$ & $\star$ & $\star$ & 0 & 0 & CT ST & VT \\
\hline 0 & 0 & $\bullet$ & - & $\star$ & TCWS & VTSW \\
\hline 0 & $\star$ & 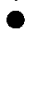 & $\star$ & $\hat{\star}$ & $\begin{array}{l}\text { LHI NC CC } \\
\text { CT ST SWP } \\
\text { NFWP }\end{array}$ & QVTSW \\
\hline$\star$ & $\star$ & $\bullet$ & 0 & 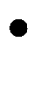 & $\begin{array}{l}\text { LHI NCSC } \\
\text { T NWS CWS } \\
\text { SWP }\end{array}$ & QVTSW \\
\hline 0 & $\star$ & 0 & 0 & 0 & $\underset{T}{\mathrm{LHI}} \mathrm{NCSC}$ & QT \\
\hline $\mathrm{O}$ & 0 & $\star$ & 0 & 0 & ST only & $\mathrm{V}$ \\
\hline & - & - & 0 & 0 & $\begin{array}{l}\text { ST CWS } \\
\text { SWS }\end{array}$ & V \\
\hline
\end{tabular}




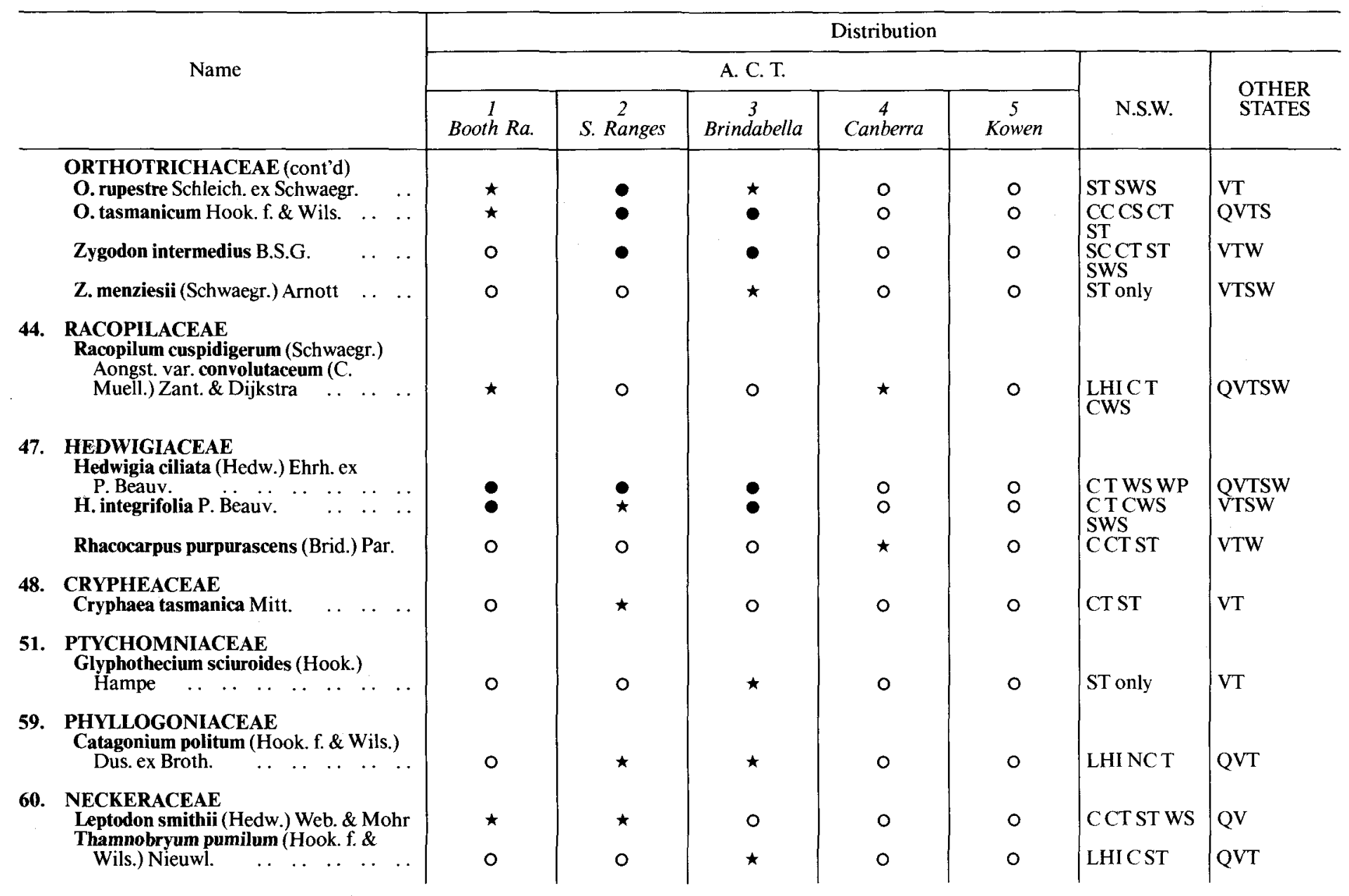


61. LEMBOPHYLLACEAE

Camptochaete arbuscula (Sm.) Reichdt. Lembophyllum divulsum (Hook. f. \&

Wils.) Lindb.

65. HOOKERIACEAE

Achrophyllum dentatum (Hook. f. \&

Wils.) Vitt \& Crosby $\ldots$.
Distichophyllum microcarpum (Hedw.) Mitt.

D. pulchellum (Hämpe) Mitt.

Sauloma tenella (Hook. f. \& Wils.) Mitt

68. HYPOPTERYGIACEAE

Hypopterygium rotulatum ( $\mathrm{Hedw}$.)

Brid.

70. FABRONIACEAE

Fabronia australis Hook.

F. brachyphylla C. Muell.

71. LESKEACEAE

Pseudoleskea imbricata (Hook. f. \&

Wils.) Broth. .. . . . . . .

72. THUIDIACEAE

Anomodon tasmanicus Broth

Thuidium furfurosum (Hook. f. \& $\ddot{\text { Wils.) }}$

Reichdt.

\begin{tabular}{|c|c|c|c|c|c|c|}
\hline 0 & 0 & - & $\star$ & 0 & LHI C CT ST & QVT \\
\hline$\star$ & 0 & - & $\star$ & $\star$ & NCSC T & QVTS \\
\hline 0 & - & - & 0 & 0 & LHI C T & VTS \\
\hline $\begin{array}{l}0 \\
\star \\
0\end{array}$ & $\begin{array}{l}0 \\
0 \\
0\end{array}$ & $\begin{array}{l}\star \\
\star \\
\star\end{array}$ & $\begin{array}{l}0 \\
0 \\
0\end{array}$ & $\begin{array}{l}0 \\
\circ \\
0\end{array}$ & $\begin{array}{l}\text { SC CT ST } \\
\text { CCT ST } \\
\text { NT ST }\end{array}$ & $\begin{array}{l}\text { VTS } \\
\text { QVTS } \\
\text { VT }\end{array}$ \\
\hline 0 & 0 & - & $\star$ & 0 & LHICT & QVTS \\
\hline - & 0 & - & - & $\star$ & $\begin{array}{l}\text { CCT ST } \\
\text { CWS SWS } \\
\text { SWP NFWP } \\
\text { NCCCST }\end{array}$ & $\begin{array}{l}\text { QVTSW } \\
\text { Q }\end{array}$ \\
\hline$\bullet$ & - & - & $\star$ & $\star$ & C T WS & VTS \\
\hline 0 & 0 & - & 0 & $\star$ & ST only & VTSW \\
\hline$\star$ & $\star$ & 0 & 0 & 0 & $\begin{array}{l}\text { LHI CT } \\
\text { NWS SWS } \\
\text { NWP }\end{array}$ & QVTSW \\
\hline $\begin{array}{l}\star \\
0 \\
0\end{array}$ & $\stackrel{\star}{\star}$ & $\begin{array}{l}\bullet \\
\star \\
\star\end{array}$ & $\begin{array}{l}\star \\
0 \\
0\end{array}$ & $\begin{array}{l}\star \\
\text { ○ } \\
0\end{array}$ & $\begin{array}{l}\text { SC NT } \\
\text { C NT ST } \\
\text { ST only }\end{array}$ & $\begin{array}{l}\text { QVTSW } \\
\text { QVT } \\
-\end{array}$ \\
\hline 0 & 0 & - & 0 & 0 & NC NT ST & VTS \\
\hline 0 & $\star$ & 0 & 0 & 0 & ST only & VT \\
\hline
\end{tabular}

T. furfurosum (Hook. f. \& Wils.)

Reichdt. var. sparsum (Hook. f. \&

Wils.) Sainsb.

T. laeviusculum (Mitt.) Jaeg.

T. subglaucinum Card.

73. AMBLYSTEGIACEAE

Acrocladium chlamydophyllum

f. \& Wils.) C. Muell. \& Broth.

Calliergonella cuspidata (Hedw.)

Loeske

\begin{tabular}{l|l}
$\circ$ & ST only
\end{tabular} 


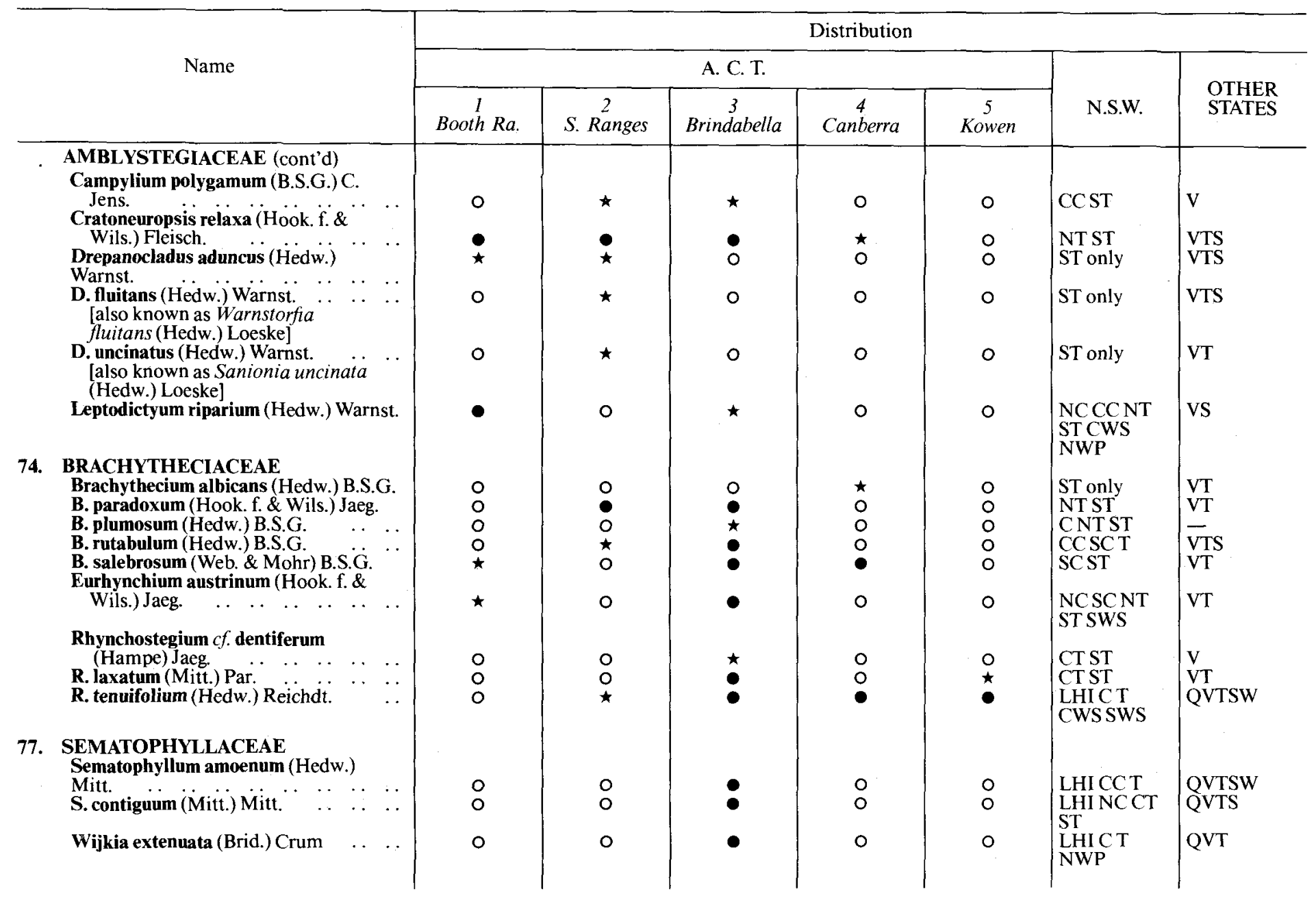


78. HYPNACEAE

Hypnum cupressiforme Hedw.

H. cupressiforme Hedw. var.

cupressiforme

H. cupressiforme Hedw. var filiforme

Brid.

H. cupressiforme Hedw. var

mossmanianum (C. Muell.) Ando

83. POLYTRICHACEAE

Atrichum androgynum (C. Muell.) Jaeg.$$
\text { var. androgynum }
$$

Pogonatum subulatum (Brid.) Brid

Polytrichastrum alpinum (Hedw.) G.L.

Smith

Polytrichum commune Hedw. ${ }^{\prime} \quad$.

$\begin{array}{lllll}\text { P. juniperinum Hedw. } & \ldots & \ldots & \ldots & \end{array}$

84. DAWSONIACEAE

Dawsonia longiseta Hampe

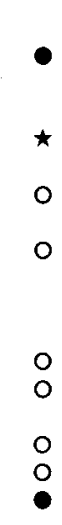

\begin{tabular}{|c|c|c|c|c|c|}
\hline - & - & - & $\bullet$ & $\begin{array}{l}\text { LHI C T WS } \\
\text { NWP }\end{array}$ & QVTSW \\
\hline$\star$ & - & $\star$ & 0 & SC ST & VT \\
\hline 0 & 0 & $\star$ & 0 & ST only & V \\
\hline O & $\star$ & 0 & 0 & ST only & VT \\
\hline $\begin{array}{l}0 \\
0\end{array}$ & $\stackrel{\bullet}{\star}$ & $\begin{array}{l}0 \\
0\end{array}$ & $\begin{array}{l}0 \\
0\end{array}$ & $\begin{array}{l}\text { LHI CCSC T } \\
\text { C T NWS }\end{array}$ & $\begin{array}{l}\text { QVT } \\
\text { QVT }\end{array}$ \\
\hline ^ & : & $\begin{array}{l}0 \\
\star \\
\bullet\end{array}$ & $\begin{array}{l}0 \\
0 \\
\star\end{array}$ & $\begin{array}{l}\text { ST only } \\
\text { NC T } \\
\text { CC SC T wS }\end{array}$ & $\begin{array}{l}\text { VT } \\
\text { QVT } \\
\text { QVTS }\end{array}$ \\
\hline o & $\star$ & 0 & 0 & $\begin{array}{l}\text { CCT ST WS } \\
\text { SWP }\end{array}$ & QVTS \\
\hline
\end{tabular}




\section{SUMMARY OF DATA}

A total of 180 species are recorded from 39 families. There are no type specimens for which the collection site is listed in the A.C.T. Species that have been attributed to the A.C.T. in Scott \& Stone (1976), but for which no specimens have been located, have not been included here.

Species diversity within the five areas described varies greatly. Kowen Forest (Division 5) contains only 34 of the listed species. This area is the smallest with less variation in habitat and has been affected by cultivation, e.g. Pinus radiata forests. The Brindabella Ranges (Division 3 ) have a great range in altitude and habitat from river flats to alpine regions and also have the greatest diversity -137 species. Although the Canberra Plain (Division 4) is most influenced by settlement, with the City of Canberra and grazing properties covering extensive areas, the moss flora is as diverse ( 89 species) as in the Southern Ranges (Division 2) (83 species) where there are greater differences in altitude and vegetation and little disturbance has occurred. This latter area is perhaps undercollected at present. The Booth Range (Division 1), which also has had little disturbance by settlement or grazing, has a surprisingly low species number ( 55 species). Again the area may be undercollected at present.

The relationships and distribution of mosses in the A.C.T. are primarily with temperate species in southern Australia, mainly Victoria and Tasmania. Of the species represented, only 69 occur also in Queensland and most of these are species that have a wider extra-Australian range. There are 35 taxa that have been collected only in the Southern Tablelands of New South Wales although all but two, Thuidium subglaucinum and Brachythecium plumosum, occur elsewhere in Australia.

\section{ACKNOWLEDGEMENTS}

Preparation of these data has depended on much help from a number of people to whom we are most grateful. Dr W.A. Weber allowed use of his unpublished preliminary list of the mosses of the A.C.T. The Directors and staff of the various herbaria have been most co-operative in allowing access to collections. Drs $\mathrm{H}$. Ochi, G.A.M. Scott, I.G. Stone and A. Touw have helped in checking determinations of many species. Professor D.G. Catcheside has provided information on a number of species, including some new records. Professors D.J. Anderson and D.G. Catcheside have assisted with suggestions for improvements to the manuscript. We are indebted to K. Cowan, Australian National University, for preparation of the map in Figure 1.

\section{REFERENCES*}

Gunn, R.H., Storey, R., Galloway, R.W., Duffy, P.J.B., Yapp, G.A. \& McAlpine, J.R. (1969). 'Lands of the Queanbeyan - Shoalhaven Area, A.C.T. \& N.S.W.' CSIRO: Land Research Series No. 24. (Melbourne.)

Learmonth, A. \& N. (1973). 'Encyclopedia of Australia.' (Frederick Warner \& Co: London.) Edn 2.

Pryor, L.D. (1954.) Plant communities. In White, H.L. (ed.), 'Canberra - A Nation's Capital.' (Angus \& Robertson: Sydney.) pp. 153-161.

Strusz, D.L. (1971). 1: 250,000 Geological Series - Explanatory Notes. CANBERRA, Australian Capital Territory \& New South Wales. (Bureau Mineral Resources: Canberra.)

*For a full reference list see Ramsay, H.P. (1984), Census of New South Wales mosses, Telopea (2)5: 455-533. 